\title{
Evolución y Características de las Publicaciones Biomédicas Peruanas, 1985 - 1993
}

\author{
JORGE ALARCÓN, JUAN MURILLO, JULIA PISCOYA, CECILIA CASTRO, CARMEN ISASI. \\ Sección de Epidemiología, Instituto de Medicina Tropical "Daniel A. Carión", U.N.M.S.M.
}

\begin{abstract}
RESUMEN
Se realizó un estudio de la evolución y pribcipales caracteristicas de los artículos científicas de investigaciones realizadas en el Perć, publicadas en revistas uacionales y extranjeras en el período 1985-1993. Durante este período se logró identificar 2412 artículos científicos publicados en 32 revistas hiomédicas nacionales, y 297 articulos aparecidos en 134 revistas extranjeras. Se observó que la mayoria de revistos médicas nacionales tiene una periodicidad irregular, siendo las revistas de sociedades cientificas las ulás coustautes. Tamujićn se observó que en 8 años el volumen de artículos científicos en revistas uacionales sólo se incrementó en $12 \%$. Además, se describen las características de los antores nacionales y extranjeros, los tipos de artículo que se pulblican $y$ las áreas temáticas. Finalmente, se hacen alcances acerca de los factores relacionados a las características y tendencias obseryadas.
\end{abstract}

Palabras claves: Investigación biomédica, Evolución, Estadistica.

\section{EVOLUTION AND CHARACTERISTICS OF PERUVIAN BIOMEDICAL PUBLICATIONS, 1985 - 1993 SUMMARY}

A study about the evolution and main characteristics of scientific articles related to rescarch conducted in Peru published both in national and international journals between $1985-1993$, was made. We identified 2412 scieltific articles published in 32 national journals, and 297 articles in 134 international journals. There was found that national journals had published sporadically, and the amount of articles increased only $12 \%$ in 8 years. In addition, the author's features, the type of scientific articles and the topics stadied, are described. Finally, sonze explanatious about the factors associated to evolution and characteristics observed, are given.

Key words: Biomedical research, Evolution, Statistics.

\section{INTRODUCCION}

La actividad cientílica de un país tiene estrecha felación con su desarrollo económico. Sin embargo. además de los recursos económicos, influyen en la producción científica otros factores, como los recursos humanos y las políticas y prioridades que cada nación establece. Esta podría ser la razón por la

\footnotetext{
Carrespondencra:

Dr. Jorge Alarcón

Departamenlo de Medicina Preventiva y Salud Pública

Faculiad de Medicna - U.N.M.S.M.

Av. Grau 755, Lima 1 - Peru

* Investigación financiada por la O.P.S. Contralo $N^{\circ}$ 162-ASC.
}

que en America Latina pese a las limituciones existentes, han existido periodos donde se logro ploducir conocimientos de calidad y originalidad. Este hecho ha motivado a estudiar las características de la producción cientifica en América Latina, con el objeto de inferir los factores dinamizadorex que deberán ser tomados en cuenta en una adecuada política de desarrollo cientifico-tecnutógico.

Entre los numerosos indicadores utilizados para estudiar la producción científica, los más difundidos son el volumen de publicaciones, la utilización de las publicacioness y la producción biomédica.

Considerando el volumen de publicaciones, América Latina se encuentra en una situación intermedia (1) coll respecto a las demás regriones. Un análisis de la producción científica en 88 
países en yías de desarrollo, mostró que México y Brasil estaban dentro de los 4 primeros lugares a nivel de la producción cientifica de dichos países, conjuntamente con la India y la antigua Yugaslavia. Estos 4 países produjeron el $52,7 \%$ de las pablicaciones científicas de este grupo de países $\left({ }^{2}\right)$. A pesar de la importancia de la producción científica de América Latina a nivel de los páses en desarrollo, dicha región está muy distante de la producción científica de los países desarrollados. En 1983, sólo el 1,14\% del total de los artículas publicados en el mundo correspondieron a América Latina ('). En 1982, América Latina produjo 34112 artículos científicos, mientras que en los EE.UU. se produjeron 135953 artículos. Pero no solamente es escasa la praducción científica en la región, sino que esta concentrada en muy pocos países. En el periodo 1973-1984 sólo 4 países (Argentina, Brasil, México y Venezuela) concentraron el $90 \%$ de todas las publicaciones Latinoamericanas. Esta tendencia se ha ido acentuando en los últimos años (").

No existen muchos estudios acerca de la utilización de la producción científica. Los pocos existentes muestran que la producción latinoamericana es muy poco citada y por ende poco utilizada por la comunidad científica internacional.

Otro indicador utilizado es la producción científica en el área de salud. Los datos existentes hasta el momento para América Latina no permiten una comparación debido a las diferentes metodologías empleadas para la recolección de la información. De otro lado, existen diferencias entre las revistas biomédicas, en particular con relación a la exigencia para aceptar y juzgar el valor de una publicación. A pesar de estos inconvenientes, según un estudio donde se tomó en cuenta 6 países de mayor producción científica en general (Argentina, Brasil, Cuba, México, Venezuela y Chile), se pueden identificar las siguientes tendencias ('):

El 55\% de artículos fueron del área de la salud.

En el periodo observado la investigación clínica ocupó el lugar principal con una tendencia al incremento, el área de ciencias básicas presentó la misma tendencia pero en menor proporción.

- La investigación en salud pública mostró una tendencia baja y estable a lo largo del periodo de análisis .

- El área temática de investigación mostró diferencias, según si las publicacjones cran en revistas nacionales o extranjeras, observándose un incremento en las publicaciones sobre ciencias básicas en las revistas internacionales, en desmedro de la proporción de investigación clínica, aunque siempre conservando esta última el primer lugar.

En el Perú, el Consejo Nacional de Ciencia y Tecnología (CONCYTEC) tiene registradas a 700 instituciones que se dedican a actividades científicas y tecnológicas, de las cuales el $32 \%$ pertenecen al sector público, el $31 \%$ al sector privado, el
$27 \%$ a uniyersidades estatales, el $7 \%$ pertenecen a universidades privadas y el $3 \%$ a instituciones internacionales $\left({ }^{3}\right)$. En esta misma institución, CONCYTEC, se registra que el número de investigadores tiende a incrementarse en los últimos años (1987 a 1990); el número de proyectos presentados también mostró un incremento notorio en 1988 y 1989 , pero disminuyeron para el año 1990. Las tres principales áreas temáticas de los proyectos registrados en el CONCYTEC en 1990 son las investigaciones en Tecnologa de Desarrollo, Ciencias Médicas y Salud, Economía y Planificación $\left({ }^{3}\right)$.

Se han becho algunos esfuerzos para evaluar el estado de to investigación científica nacional a través de las publicaciones biomédicas. Así, en el marco del "Seminario Nacional de Priorización de la Investigación Científica en Salud", se planteó a las publicaciones biomédicas como indicador válido para evaluar la producción científica. En este evento. se presentó un análisis de las características de las publicaciones periódicas peruanas en el periodo 1979-1982, donde se estudiaron tanto los trabajos aparecidos en la literatura nacional como los estudios sobre el Perú publicados en el extranjero (4). Sin embargo, en esta primera aproximación se consideró un número limitado de variables, lo cual no permitió realizar un análisis profundo de la problemática de las publicaciones nacionales. Otra aproximación al estudio de las características de las publicaciones nacionales es el análisis de los estudios epidemiológicos publicados en 5 revistas médicas nacionales en el periodo 1979-1983 ( ${ }^{5}$ ). Como parte de este estudio, se realizó una descripción global de las características de los trabajos publicados en las revistas seleccionadas; pero sólo comprendió al $20 \%$ de las publicaciones del periodo y no tomó en cuenta los estudios publicados en el extranjero. De otro lado, las definiciones operacionales utilizadas son poco precisas para una descripción adecuada de las características de las investigaciones publicadas.

Fuera de estas primeras aproximaciones que describen algunas de las características de las publicaciones médicas ciel periodo 1979-1983, no existen estudios que hayan realizado una descripción y análisis de las características de las investigaciones biomédicas publicadas en las principales revistas médicas nacionales y extranjeras, Io cual es una limitación importante tanto para la evaluación de la producción científica nacional, como para la elaboración de políticas de desarrollo de la investigación en el país. Asimismo, no se han rẹalizado estudios para calificar las publicaciones médicas que se editan en el país, por to cual no se puede saber cuántas de ellas cumplen con todos los requisitos de una publicación científica.

De ocro lado, existen divergencias en cuanto a la validez de algunas metodologías de estudio de las publicaciones biomédicas. Los problemas radican en que algunos indicadores utilizados en investigaciones desarrolladłs en América Latina, son elaborados en base a criterios originados en países desarrollados. Algunos de estos indicadores se basan en la 
proporción en que aparecen investigaciones de América Latina en bases de datos de países industrializados. Estos indicadores ban sido seriamente cuestionados en los últimos años, en la medida en que hay muchas revistas científicas de paises poco desarrollados que no son considerados en los registros que elaboran los países industrializados $\left({ }^{6}\right)$. Todo ello nos muestra que un análisis de la producción científica de nuestra región, a través de las investigaciones publicadas en la literatura, tiene que dar prioridad al análisis de las publicaciones locales.

El presente estudio pretende contribuir a la comprensión de algunos de estos aspectos a partir del análisis de indicadores sencillos. Su objetivo es describir la evolución y las principales características de los artículos científicos publicados en las revistas medicais nacionalés y extranjeras en el periodo 1985-1993.

\section{MATERIAL Y METODOS}

Se hizo un listado de las revistas periódicas nacionales relacionadas a salud aparecidas en el periodo enero 1985-diciembre 1993, así como de las Tevistas biomédicas extranjeras, correspondientes al mismo periodo, donde se publicaron articulos referentes a investigaciones realizadas en el Perí. Con este fin se consultó el centro de documentación de la OPS y los ficheros de las bibliotecas de las Facultades de Medicina de la UNMSM $y$ de la UPCH. Respecto a las revistas nacionales, en los casos en que la colección de la revista no sé encontró completa, se contactó con algunos editores o sociedades científicas para tener acceso a sus respectivos archivos. En los casos de las revistas extranjeras, se tomó como referencia los artículos sobre el Perú que aparecen en las bases de datos MEDLINE y LILACS para el periodo de estudio.

Para una selección de las publicaciones, sujeto de estudio, se consideró como revista médica o revista de sálud a toda publicación que tenga como línea editorial la difusión de un campo específico de las ciencias de la salud. No se tomó en cuenta para efectos de este estudio las publicaciones periódicas no médicas en que aparecen investigaciones en salud.

Respecto a las revistas nacionales, en los casos en que la colección de la revista no se encontró completa, se contactó con algunos editores o sociedades científicas para tener acceso a sus respectivos archivos. De esa forma se identificaron 51 publicaciones periódicas nacionales y 134 extranjeras. Se seleccionaron para el estudio, 32 publicaciones nacionales y todas las publicaciones extranjeras. Las revistas nacionales se seleccionaron en base a los siguientes criterios de inclusión:

- Mayor frecuencia de publicación.

- Que representase una tendencia importante en la producción científica nacional.

- Que contenga artículos originales.

- Que tenga circulación tracional
En cada caso se estudió las características de la revista, de los autores y de la publicación. Como características de la revista se tomaron en cuenta, el nombre, la fecha de publicación y el lugar de publicación (nacional o extranjera): Respecto a los autores se estudió el número de autores, el lugar de residencia del autor principal y su calificación protesional (profesión). Del artículo se analizó el tipo de escrito, el área y temas específicos.

El manejo de la información se realizó mediante el programa de cómputo Fox Pro 20. El análisis estadístico fue descriptivo y se realizó mediante el programa estadístico SPSS-PC 4.0.

\section{Características de las revistas \\ RESULTADOS}

La periodicidad de las revistas bionédicas peruanas en el lapso 1985-1993 se caracterizó por no mantener un número constante de publicaciones por año e incluso por haber años en los que no se publicó. En la Tabla $N^{0} 1$ se puede observat el compottatniento de las 32 publicaciones seleccionadas. Se aprecia que las revistas biomédicas más regulares fueron las ugue pertenecen o estár ligadas a Sociedades Científicas.

Entre enero de 1985 y diciembre de 1993 se han publicado un total de 2412 artículos cienúficos, en sus diferemes modalidades, en revistas biomédicas nacionales. En este mismo perífo fueron publicados 297 artículos acerca de investigaciones realizadas en el Peru, en revistas biomédicas exuanjeras. Esla producción representa un total de 2709 artículos publicados, $89 \%$ en revistas nacionales y $11 \%$ en revistas extranjeras.

E) $75 \%$ de los artículos publicados en revistas nacionales aparecieron en 14 de las 32 revislas analizadas en este extudio. La Tabla $N^{\circ} 2$ muestra la lrecuencia de artículos publicados ea las revistas seleccionadas.

Las publicaciones en revistas extranjeras han sido más frecuentes en 22 de las 134 revistas extrarjeras que publicaron algún artículo acerca de estudios realizados en el Perú. En las otras revistas los artículos han tenido una aparición esporádica. En la Tábla $N^{0} 3$ se muestra la relación de las 22 revistas extranjeras que con mayor frecuencia publicaron artículos referentes a investigaciones realizadas en el Perú en el periodo do estudio.

El número de publicaciones en revistas nacionales ba mostrado muy pocas yariaciones, la mediana fue de 268 artículos por año, con un mínimo de 242 y un máxino de 303 . Es notorio el incremento en el año 1991 y 1992. En el año 1993 hubo una disminución de las publicaciones del orden del $9.6 \%$ con respecto al año anterior (ver Figura $N^{c} 1$ ).

Los artículos aparecidos on las revistas extranjeras han tenido una tendencia semejance a las publicacioncs nacionales, aunque la proporción de artículos cientificon yue se publican en revistas biomédicas extranjeras en relación al total de la producción ha tendido a incrementarse. En los años 1989 y 1991 la proporción fue mayor que la tendencia (ver Fig. $N^{0} 1$ ) 
TABLA 1.- Periodicidad de las revistas biomédicas nacionales seleccionadas. Perú, 1985-1993.

\begin{tabular}{|c|c|c|c|c|c|c|c|c|c|}
\hline Revista & 85 & 86 & 87 & 88 & 89 & 90 & 91 & 92 & 93 \\
\hline Diagnóstico & 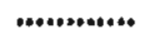 & enenentuene. & .encen....... & 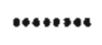 & œ... & .....* & ...et." & $\cdots$ & $\cdots$ \\
\hline Revisla de la Sanidad de las Fuerzas Policiales & $\bullet$ & $*$ & " & $*$ & $\because$ & $\because$ & - & $\cdots$ & $\bullet$ \\
\hline Acta Médica Peruana & $\cdots$ & $*$ & $\bullet$ & - & & & $\because$ & $\cdots$ & \\
\hline Revista Peruana de Gastroesterología & 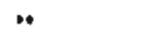 & $\ldots$ & $\cdots$ & $\cdots$ & $\cdots$ & $\cdots$ & $\bullet$ & • & $\cdots$ \\
\hline Revista de Neuro-Psiquiatría & 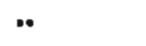 & $\ldots$ & ... & nat. & $\cdots$ & *. & $\cdots$ & $\cdots$ & $\cdots$ \\
\hline Medicamentos y Salud Popular & & -- & $\ldots$ & wast* & • & $\cdots$ & ... & $\cdots$ & ... \\
\hline Revista Peruana de Ginecología y Obstetricia & - & • & - & ** & .. & - & $\bullet$ & • & - \\
\hline Revista Médica Herediana & & & & & & $*$ & $\cdots$ & $\cdots$ & $\bullet$ \\
\hline Cirujano & - & 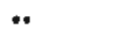 & 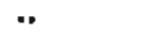 & & - & & $\bullet$ & - & \\
\hline Salud Popular & & $*$ & $\cdots$ & 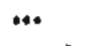 & $\bullet$ & $\bullet$ & - & - & $\cdot$ \\
\hline Revista del Cuerpo Mélico GAI (*) & & & & • & - & - & & - & \\
\hline Revista Peruana de Epidemiología & & $*$ & - & & & 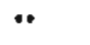 & $\bullet$ & $*$ & $\cdot$ \\
\hline Anales de Salud Mental & - & - & $\cdot$ & * & - & • & - & & \\
\hline Acta Médica del Hospital Militar & - & • & - & • & • & & & & \\
\hline Acta Cancerológica & & & & • & - & • & & & \\
\hline Revirta Peruana de Medicina Tropical & & & & & & & " & - & 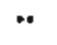 \\
\hline Revjsta Peruana de Oftalnología & & $\cdots$ & "• & * & 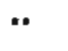 & & - & • & - \\
\hline Revinla Médica Peruana & & & & & $\bullet$ & $\cdot$ & • & $\bullet$ & $\cdot$ \\
\hline Revista Oftalmológjea & $\ddot{*}$ & $*$ & $\ddot{*}$ & $\bullet$ & - & & & - & $\cdot$ \\
\hline Psicoactiva & & & ... & - & - & - & - & - & \\
\hline Boletín de la Soc. Peruana de Medicina Inkerna & - & & $\ddot{*}$ & & & & & $* \cdots$ & $\cdots$ \\
\hline Revista de Farmacología y Terapéutica ' & & & & & & & $\bullet$ & $\cdots$ & - \\
\hline Revista del Instituto Nacional del Niño & - & & & • & & $\cdot$ & & $\cdot$ & \\
\hline Revista de la Soc. Peruana de Tisiología, (**) & & & & . & & & 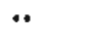 & $\cdot$ & \\
\hline Revista de lá Academia Peruana de Cirugía & & - & & - & - & & & & - \\
\hline Boletín del' TMT-DAC-UNMSM & - & $\ddot{*}$ & & & & & & & \\
\hline Actal Andiná & & & & & & & & •* & •• \\
\hline Galeno & ate.... & $\cdots$ & & & & & & & \\
\hline Boletín Peruano de Parasitología & & & & - & & & & & \\
\hline Analer de la Facultad de Medicima -LNMSM & & & & & - & & & & \\
\hline Salud Pública & & & & & & & & & $\cdots$ \\
\hline Revisla Peruana de Cardiologia & & & & & & & & & - \\
\hline
\end{tabular}

(•) Caula punto representa un múnzero de la revista.

(*) Revista del Cuerpo Mćdico del Hospital IPSS Guillermo Almerara

(**) Sociedad Peruana de Tisiología, Neumología y Enfernedades del Tóras. 
TABLA 2.- Artículos Científicos publicados en Revistas Biomédicas Nacionales. Perú, 1985- 1993.

\begin{tabular}{|c|c|c|}
\hline Revista & $N^{\circ}$ & $\%$ \\
\hline Diagnóstico & 323 & 13.4 \\
\hline Revista de la Sanidad de las Fuerzas Policjales & 212 & 8,8 \\
\hline Acta Médica Peruana & 167 & 6.9 \\
\hline Revista Pcruana de Gastroenterología & 139 & 5,8 \\
\hline Revista Neuro-Psiquialría & 126 & 5,2 \\
\hline Medicamentos y Salud Popular & 117 & 4,9 \\
\hline Revista Peruana de Ginecología y Onstetricia & 108 & 4,5 \\
\hline Revista Méclica Herediana & 103 & 4,3 \\
\hline Cirujano & 97 & 4,0 \\
\hline Salud Popular & 94 & 3,9 \\
\hline Revista del Cuerpo Médico del Hospital IPSS* & 85 & 3,5 \\
\hline Revista Peruana de Epidemiología & 80 & 3.3 \\
\hline Anales de Salud Mental & 79 & 3,3 \\
\hline Acla Mélica def Hospital Militar & 73 & 3,0 \\
\hline Actá Cancerológica & 67 & 2,8 \\
\hline Revista Peruana de Medicina Tropical & 62 & 2,6 \\
\hline Revista Pecuana de Oftalnología & 60 & 2,5 \\
\hline Revista Médica Peruana & 57 & 2,4 \\
\hline Revista del Inalituto Nacional de Oftalmología & 57 & 2,4 \\
\hline Psicoactiva & 43 & 1,8 \\
\hline \multicolumn{3}{|l|}{ Boletín de la Sociedad Peruana de } \\
\hline Medicina Interna & 42 & 1,7 \\
\hline Revisca de Farmacología y Terapéutica & 38 & 1,6 \\
\hline Revista dol Instituto Nacional del Niño & 32 & 1,3 \\
\hline Revista de la Sociedad Peruana de Tisiología:** & * 30 & 1,2 \\
\hline Revista de la Academia Peruana de Cirugía & 30 & 1,2 \\
\hline Boletín del Inscituto de & & $\therefore$ \\
\hline Medicina Tropical - UNMSM & 21 & 0,9 \\
\hline Acca Andina & 20 & 0,8 \\
\hline Galeno & 17 & 0,7 \\
\hline Boletín Peruana de Parasitologia & 11 & 0,5 \\
\hline Analer de la Faculiad de Medicina & 9 & 0,4 \\
\hline Salucl Pública & 8 & 0.3 \\
\hline Revisla Peruana de Cardjología & 5 & 0.2 \\
\hline Total & 2412 & 100,0 \\
\hline
\end{tabular}

(*) Revista del Cuerpo Médico del Hospital IPSS Guillerno Almenara

(**) Revista de la Sociedarl Peruana de Tisiologia, Neumología y Enfermedades dé Tórax

\section{Características de los autores}

En total se registraron 7605 autores, de los cuales $36 \%$ figuran como autores principales. La proporción de autores que aparecen en publicaciones nacionales fue de $82 \%$. Entre los autores que aparecen en las publicaciones nacionales, el $85 \%$ reside en Lima, la capital del país, el $8.3 \%$ en provincias y el $6,7 \%$ en el exuranjero. Entre los autores que figuran en arlículos de publicaciones extranjeras, el 52,8\% reside en Lima, el 3,7\% en provincias y el $43,5 \%$ en el extranjero.
TABLA 3.- Revistas biomédicas extranjeras con mayor publicación de artículos científicos nacionales. Perú, 1985 - 1993

\begin{tabular}{|c|c|c|}
\hline Revista & $\therefore \%$ & $\mathscr{\%}$ \\
\hline Bolelín de la Oncina Sanitaria Pananjericana & 16 & 5.4 \\
\hline Medicine and Hygjere & 15 & 5.1 \\
\hline The Lancel & 10 & 3.4 \\
\hline Anerican Journal of Clinical Nutrition & 9 & 3.0 \\
\hline The Journal of Infectious Disease & 8 & 2,7 \\
\hline Socjal Science of Merlicine & 8 & 2.7 \\
\hline Tropical Medicine and Parasitology & 7 & 2.4 \\
\hline \multicolumn{3}{|l|}{ Transactions of the Royal Society of } \\
\hline Tropical Medic Hygiene & 7 & 2.4 \\
\hline Archives of Andrology & 7 & 2.4 \\
\hline Journal of Clinical Microbiology & 7 & 2.4 \\
\hline Seminars of Surgical Oncology & 7 & 2,4 \\
\hline Studies of the Tamily Planning & 6 & 2,0 \\
\hline Journal of Pediatric Gastroenterology Nutricion & 6 & 2.0 \\
\hline American Journal of Epideniology & 4 & 1.3 \\
\hline Journal of Mudical Entonology & 4 & 1,3 \\
\hline Journat of Ethnopharmacology & 4 & 1.3 \\
\hline Mycoser & 4 & 1.3 \\
\hline Pediatrics ln[ectuous Diseases Journa] & 4 & 1.3 \\
\hline Americat Journal of Physic and Anthropulagy & 4 & 1.3 \\
\hline Prog-Clin-Biol-Res & 4 & 1,3 \\
\hline Archivos Latinoamericanos de Nutricion & 4 & 1.3 \\
\hline American Journal of Gastroenterology & 4 & 1,3 \\
\hline Resto do Publicaciones & 148 & 49,8 \\
\hline Tolal & 297 & 100,0 \\
\hline
\end{tabular}

Los autores extranjeros que participan en publicaciones de estudios reálizador en el Perú en revistás biomédicas nacionales, son mayormente norleamericanos, $2 \%$ del total de autores y $29,8 \%$ del tolal do autores exiranjesos (Tabla $N^{\prime} 4$ ).

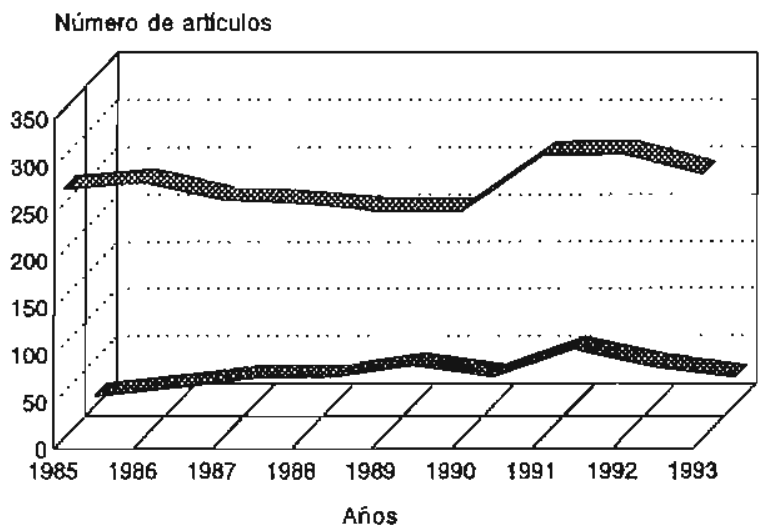

Fig. 1.- Frecuencia de Arlículos Científicos Peruanos Publicados en Revistas Biomédicas Nacionales (línea superior) y Extranjeras (línea inferior), 1985 - 1993 
TABLA 4.- Procedencia de los autores de revistas biomédicas nacionales. Perú, 1985 - 1993.

\begin{tabular}{lrr}
\hline Procedenciá & $N^{\circ}$ & $\%$ \\
\hline Perú & 5816 & 93,3 \\
Estados Unidos & 124 & 2,0 \\
España & 55 & 0,9 \\
Chile & 35 & 0,6 \\
Cuba & 32 & 0,5 \\
Argentina & 28 & 0,4 \\
México & 19 & 0,3 \\
Japón & 19 & 0,3 \\
Colombia & 18 & 0,3 \\
Inglaterra & 16 & 0,3 \\
Brasil & 15 & 0,2 \\
Jtalia & 7 & 0,1 \\
Canadá & 1 & 0,0 \\
Otros países Europeos & 24 & 0,4 \\
Otros países Latinoamericanos & 20 & 0,3 \\
Otros paises Asiáticos & 3 & 0,0 \\
& & \\
Total & 6232 & 100,0 \\
\hline
\end{tabular}

(*) Sin información de procedencia: 74 autores

TABLA 5. Procedencia de los autores que publican en revistas biomédicas extranjeras. Perú, $1985-1993$

\begin{tabular}{lrr}
\hline Procedencia & $N^{\circ}$ & $\%$ \\
\hline Perú & 725 & 56,5 \\
Estados Unidos & 429 & 33,4 \\
Canadá & 30 & 2,3 \\
Francia & 23 & 1,8 \\
İalia & 20 & 1,6 \\
Alemania & 17 & 1,3 \\
Inglaterra & 15 & 1,2 \\
Holanda & 7 & 0,5 \\
Austria & 7 & 0,5 \\
Suecia & 4 & 0,3 \\
Bélgica & 3 & 0,2 \\
Bolivia & 2 & 0,2 \\
Finlandia & 1 & 0,1 \\
Total & 1283 & 100,0 \\
\hline
\end{tabular}

(*) Sin información de procedencia: 16 autores
El $33 \%$ de los autores que publican artículos referentes a estudios realizados en el Perú en revistas biomédicas extranjoras, fueron de nacionalidad norteamericana. Estos autores a su vez representan el $76,9 \%$ de los autores extranjeros en el presente estudio.

Los departamentos del Perú donde residen los autores de provincias que han publicado en reyistas biomédicas nacionales son: en primer lugar La Libertad y en segundo lugar Arequipa. En conjunto ambos departamentos tienen un $56,8 \%$ del total de la producción de los autores del interior del país. El resto de la producción bibliográfica se distribuye en los 17 departamentos restantes, tal como se puede observar con los datos presentados en la Tabla $\mathrm{N}^{\circ} 6$.

TABLA 6.- Autores de provincias que publican en revistas biomédicas nacionales según el departamento de residencia. Perú, $1985-1993$

\begin{tabular}{|c|c|c|}
\hline Departamento & $N^{\circ}$ & $\%$ \\
\hline La Libertad & 196 & 38,0 \\
\hline Arequipa & 97 & 18,8 \\
\hline Cusca & 36 & 7.0 \\
\hline Loreto & 27 & 5,2 \\
\hline Piura & 26 & 5,0 \\
\hline Ancash & 25 & 4.8 \\
\hline Lambayeque & 21 & 4,1 \\
\hline Puno & 18 & 3,5 \\
\hline Ica & 11 & 2,1 \\
\hline Ucayali & 9 & 1,7 \\
\hline Cajamarca & 8 & 1.6 \\
\hline Cerro de Pasco & 8 & 1,6 \\
\hline San Martín & 7 & 1,4 \\
\hline Apurímac & 6 & 1.2 \\
\hline Junín & 5 & 1,0 \\
\hline Tumbes & 4 & 0,8 \\
\hline Abancay & 4 & 0,8 \\
\hline Madre de Dios & 3 & 0.6 \\
\hline Huánuco & 2 & 0.4 \\
\hline Ayacucho & 2 & 0,4 \\
\hline Tacna & 1 & 0,2 \\
\hline Total & $\$ 16$ & 100,0 \\
\hline
\end{tabular}

(*) Sin información del departamento: 38 autores 
Los Departamentos donde residen los autores de provincias que aparecen en revistas biomédicas extragjeras son: La Libertad y Arequipa, que en conjunto representan el $77 \%$, (ver Tabla No 7)

El número de autores yaría según se trate de revistas biomédicas nacionales o extranjeras. En las revistas nacionales, el $41 \%$ de publicaciones tiene un autor; en las extranjeras esta proporción fue sólo del 13,5\%. Esta relación se mantiene a través del tiempo; aunque la tendencia hacia autores únicos es a incrementarse en el caso de revistas biomédicas nacionales, y a disminuir en las exiranjeras.

La mayoría $(94 \%)$ de los autores de publicaciones en revistas biomédicas nacionales tuvo la calificación profesional de médico, le siguen los biólogos con un porcentaje menor $(3,1 \%)$; otros profesionales como los científicos sociales tienen una escasa presencia en las publicaciones biomédicas. Esta composición varía en el tempo en las publicaciones nacionales, obseryándose un incremento en la participación de autores no médicos en los últimos dos años (Tabla $\mathbb{N}^{\circ} 8$ ).

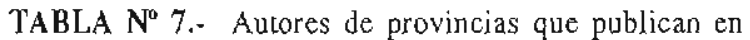
revistas biomédicas extranjeras según el departameato de residencia. Perú, 1985 - 1993

\begin{tabular}{lrr}
\hline Departamento & N & $\%$ \\
\hline La Libertad & 26 & 54,2 \\
Arequipa & 11 & 22,9 \\
Cajamarca & 2 & 4,2 \\
Loreto & 2 & 4,2 \\
Lambayeque & 2 & 4,2 \\
San Martín & 1 & 2,1 \\
Ucayaji & 1 & 2,1 \\
Pjura & 1 & 2,1 \\
Cerro de Pasco & 1 & 2,1 \\
Ayacucho & 1 & 2,1 \\
& & \\
Total & 48 & 100,0 \\
\hline
\end{tabular}

TABLA 8.- Profesionales que publican en revistas biomédicas nacionales por años. Perú 1985-1993

\begin{tabular}{|c|c|c|c|c|c|c|c|c|c|c|c|}
\hline \multirow[b]{2}{*}{ Profesión } & \multicolumn{9}{|c|}{$A \bar{n} 0$} & \multirow[b]{2}{*}{ Tolal } & \multirow[b]{2}{*}{$\%$} \\
\hline & 1985 & 1986 & 1987 & 1988 & 1989 & 1990 & 1991 & 1992 & 1993 & & \\
\hline Médico & 628 & 717 & 626 & 534 & 622 & 559 & 706 & 700 & 599 & 5691 & 93,7 \\
\hline Biólogo & 16 & 34 & 7 & 23 & 2 & 5 & 23 & 36 & 42 & 188 & 3,1 \\
\hline Psicólogo & 2 & 4 & 13 & 15 & 9 & 4 & - & 7 & 4 & 58 & 1,0 \\
\hline Nutricionisla & . & . & 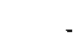 & 6 & - & - & 3 & . & - & 9 & 0.1 \\
\hline Antropólogo & - & - & 3 & 2 & 1 & 1 & 2 & 2 & 4 & 15 & 0.2 \\
\hline Socióluga & . & - & 1 & 2 & - & 2 & - & 3 & 2 & 10 & 0.2 \\
\hline Enfermera & 4 & - & 1 & 6 & - & 3 & - & 3 & 3 & 20 & 0,3 \\
\hline Odantólogo & - & 1 & 1 & - & 1 & - & - & - & - & 3 & 0,0 \\
\hline Veterinaria & - & 1 & - & 1 & - & - & 4 & 1 & 8 & 15 & 0,2 \\
\hline Economista & . & . & . & 1 & . & - & 1 & 1 & 4 & 7 & 0,1 \\
\hline Ingeniero & . & . & - & 1 & . & - & 3 & 1 & 1 & 6 & 0,1 \\
\hline Educador & - & . & . & - & 2 & - & - & . & - & 2 & 0,0 \\
\hline Historiador & . & - & - & - & - & - & . & 1 & - & 1 & 0,0 \\
\hline Quinico-Farmacéutico & 1 & - & - & - & - & . & - & - & 3 & 4 & 0,1 \\
\hline Tecnólogo Médjea & . & 6 & - & 3 & 3 & - & 3 & 6 & 16 & 37 & 0,6 \\
\hline Teólogo & . & - & . & . & . & 1 & - & . & . & 1 & 0,0 \\
\hline Comunicsdor Social & - & - & - & - & 3 & . & . & - & . & 3 & 0.0 \\
\hline Estadístico & - & 1 & 1 & - & - & - & - & - & - & 2 & 0,0 \\
\hline Demógrafo & - & - & - & - & - & - & - & - & 1 & 1 & 0,0 \\
\hline Asistenta Sacial & - & - & - & - & - & - & - & - & 1 & 1 & 0,0 \\
\hline Total & 651 & 764 & 653 & 594 & 643 & 575 & 745 & 761 & 688 & 6074 & 100,0 \\
\hline
\end{tabular}

(*) Sin información de profesioñ : 232 autores. 
Tabla N 9. Profesiomales que publican en revistas biomédicas extranjeras por affos. Pená, 1985 - 1993

\begin{tabular}{|c|c|c|c|c|c|c|c|c|c|c|c|}
\hline \multirow[b]{2}{*}{ Profesion } & \multicolumn{9}{|c|}{ Año } & \multirow[b]{2}{*}{ Total } & \multirow[b]{2}{*}{$\%$} \\
\hline & 1985 & 1986 & 1987 & 1988 & 1989 & 1990 & 1991 & 1992 & 1993 & & \\
\hline Médico & 22 & 55 & 89 & 117 & 114 & 118 & 232 & 183 & 117 & 1047 & 87,0 \\
\hline Biólogo & 4 & 1 & 4 & 7 & 19 & 12 & 34 & 21 & 29 & 131 & 10.9 \\
\hline Psicólogo & - & - & - & - & - & 1 & - & - & - & 1 & 0.1 \\
\hline Antropólogo & - & 1 & 1 & 1 & 1 & - & 2 & - & - & 6 & 0.5 \\
\hline Sociólogo & - & - & 1 & 2 & - & 2 & - & 3 & 2 & 10 & 0,2 \\
\hline Enfermera & - & - & - & - & - & - & 1 & - & - & 1 & 0,1 \\
\hline Odontólogo & - & - & - & - & 2 & - & - & - & - & 2 & 0,2 \\
\hline Velerinario & - & - & - & 2 & - & - & - & - & - & 2 & 0.2 \\
\hline Economista & - & 2 & 4 & - & - & - & - & - & - & 6 & 0.5 \\
\hline Historiador & - & - & - & - & - & - & 1 & - & - & 1 & 0,1 \\
\hline Quimico-Farmacéutico & - & - & 1 & - & - & - & - & - & - & 1 & 0,1 \\
\hline Tecnójogo Médico & - & - & - & - & - & 2 & - & - & - & 2 & 0,2 \\
\hline Total & 26 & 59 & 100 & 129 & 136 & 135 & 270 & 207 & 148 & 1210 & 100,0 \\
\hline
\end{tabular}

(*) Sin información de prolesión: 89 autores

En las publicaciones en revistas extranjeras también la mayoría de los autores fueron médicos $(87,4 \%$ ), le siguieron las otras profesiones con porcentajes muy bajos. La tendencia en el tiempo también ha sido el incremento de los autores de profesiones no médicas (Tábla $N^{\circ} 9$ ).

\section{Características del artículo científico}

El tipo de artículo má: frecuente en revistas bioméclicas nacionales fue el "trabajo original" (54\%) y le siguió con un porcentaje menor la presentación de casos, el ensayo y la revisión de lemas. Las otras formas y en especial las carlas al editor fucron muy escasas. En las revistas biomédicas extranjeras el $87 \%$ de publicaciones, $28 \%$ más que las nacionales, fueron trabajos originales, le siguieron con una frecuencia menor las cartas al cditor $y$ los editoriales.

La proporción de artículos origrinales en las revistas nacionales analizadas tuvo una amplia variación. La proporción más baja fue de $6,8 \%$ y la más alta de $81,8 \%$. El $50 \%$ de revistas publica entre 42 y $70 \%$ de artículos originales; un $25 \%$ de revislas publica menos del $42 \%$ y otro $25 \%$ publica más del $70 \%$ de arlículos originales. En la Tabla $\mathrm{N}^{\circ} 10$ se puede observar que las revistas con más de $70 \%$ de artjculos originales son en su mayoría boletines o anuarias de instituciones académicas: en cambio en las revistas con menos del $42 \%$ de artículos originales se encuentran muchas que son de un carácter de divulgación dirigidà a un público no especializado.

Las áreas de estudio a las que pertenecieron los artículos publicados en revistas biomédicas nacionales fueton en su mayoría las clínico-quirúrgicas y la epidemiologra, ambos representan el $85 \%$ de artículos publicados. La tendencia en el tiempo se puede observar en la Tabla $N^{\circ} 11$. En las revistas biomédicas extranjeras, la mayoría de artículos científicos correspondieron a las áreas de clínico-quirúrgicas, epidemiología y las ciencias básicas, que suman el $87 \%$ de los artículos publicados. La tendencia en el tiempo se puede observar en la Tablid $\mathrm{N}^{\circ} 12$. 
TABLA 10.- Artículos Científicos originales publicados en Reviștas Biomédicas Nacionales. Perú, 1985 - 1993.

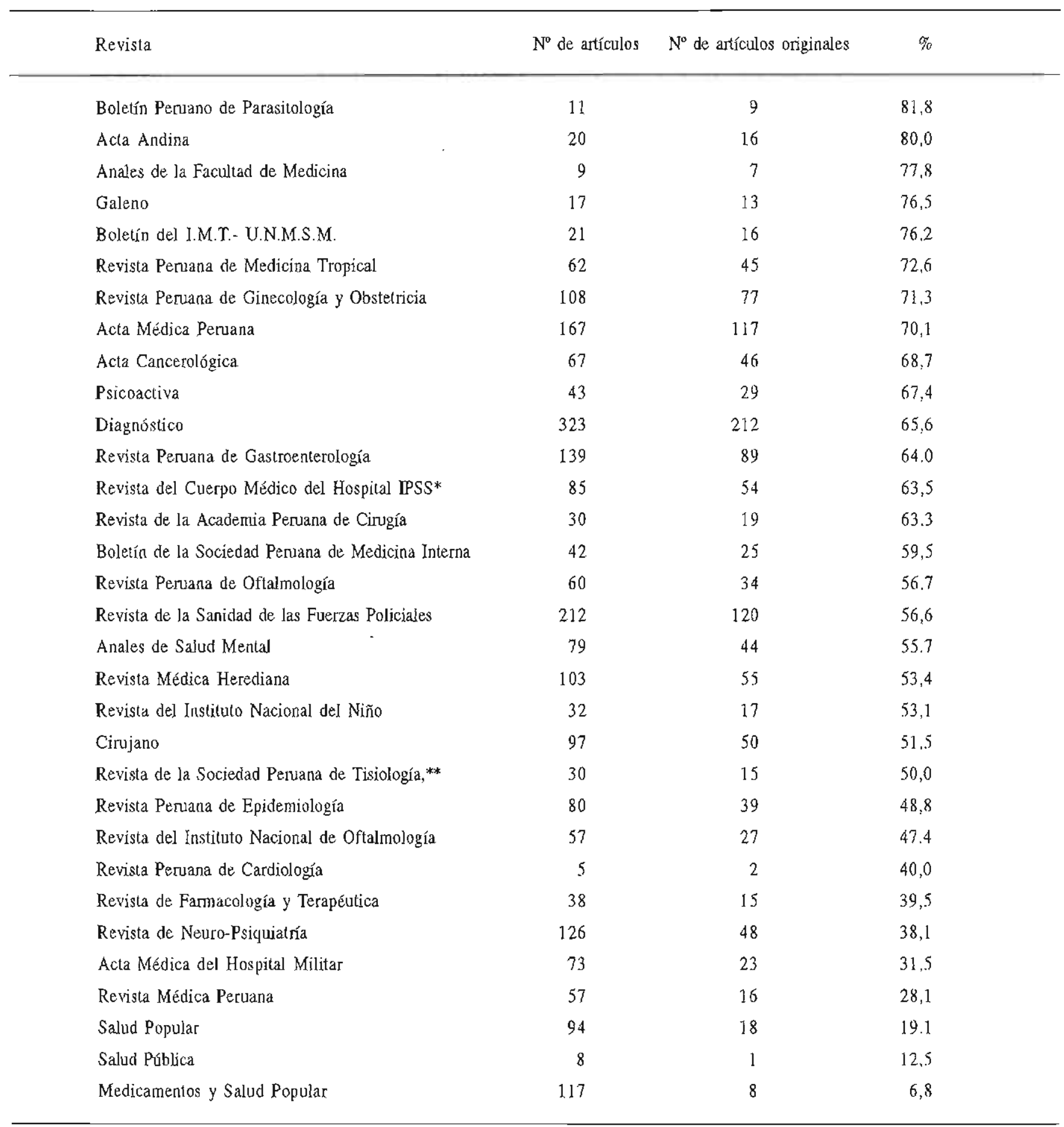

(*) Revista del Cuerpo Médico del Hospital IPSS Guillermo Almenara

(**) Revista de la Sociedad Peruara de Tísiología Neumolagía y Enfermedades del Tórax. 
TABLA 11.- Areas de estudio en las publicaciones de revistas biomédicas nacionales por años. Perú, $1985-1993$

\begin{tabular}{|c|c|c|c|c|c|c|c|c|c|c|c|}
\hline \multirow[b]{2}{*}{ Area de estudio } & \multicolumn{9}{|c|}{ Año } & \multirow[b]{2}{*}{ Total } & \multirow[b]{2}{*}{$\%$} \\
\hline & 1985 & 1986 & 1987 & 1988 & 1989 & 1990 & 1991 & 1992 & 1993 & & \\
\hline Clínico reparativa & 193 & 205 & 184 & 167 & 176 & 166 & 195 & 207 & 176 & 1669 & 69,2 \\
\hline Epidemiológico & 26 & 21 & 18 & 14 & 21 & 40 & 59 & 50 & 51 & 300 & 12,4 \\
\hline Medicina Preventiva y $S,\left(^{*}\right)$ & 10 & 19 & 30 & 33 & 22 & 26 & 15 & 11 & 10 & 176 & 7,3 \\
\hline Tecnologias aplicadas (**) & 4 & 1 & 2 & 4 & 4 & - & 2 & 1 & 1 & 19 & 0,8 \\
\hline Ciencias Bussicas & 8 & 11 & 6 & 10 & 3 & 2 & 8 & 15 & 25 & 88 & 3,6 \\
\hline Educación Médicu & 3 & 3 & - & 5 & 2 & 1 & 7 & 3 & 2 & 26 & 1,1 \\
\hline Etro-medicina & 6 & 1 & 1 & 5 & - & - & 2 & 2 & 2 & 19 & 0.8 \\
\hline Fistoria de la Medicina & 8 & 7 & 7 & 2 & 2 & 2 & 9 & 8 & 7 & 52 & 2,2 \\
\hline Economia en Salud & - & - & - & - & - & - & 1 & - & 5 & 6 & 0,2 \\
\hline Etica y Deontología Médica & 7 & 3 & 2 & 4 & 2 & 3 & - & 4 & 1 & 26 & 1,1 \\
\hline Políticas de desarro[]o (***) & 1 & 1 & 2 & 1 & - & 1 & 1 & - & 1 & 8 & 0,3 \\
\hline Epistemología & - & - & - & - & - & 1 & - & - & - & 1 & 0,0 \\
\hline Salud Ambiental & - & - & - & 4 & - & - & - & 1 & - & 5 & 0,2 \\
\hline Educación en Salud & - & 1 & 2 & 1 & 10 & - & 2 & 1 & - & 17 & 0,7 \\
\hline Total & 266 & 273 & 254 & 250 & 242 & 242 & 301 & 303 & 281 & 2412 & 100,0 \\
\hline
\end{tabular}

(*) Medicina Preventiva y Salud Pública

(**) Tecnologias aplicadas al diagnóstico y tratamiento

(**) Polícicas de desarrollo de investigación en salud

Tahla $N^{\circ}$ 12. Areas de estudio en las publicaciones de revistas biomédicas extranjeras por años. 1985-1993

\begin{tabular}{|c|c|c|c|c|c|c|c|c|c|c|c|}
\hline \multirow[b]{2}{*}{ Area de estudia } & \multicolumn{9}{|c|}{ Año } & \multirow[b]{2}{*}{ Total } & \multirow[b]{2}{*}{ to } \\
\hline & 1985 & 1986 & 1987 & 1988 & 1989 & 1990 & 1991 & 1992 & 1993 & & \\
\hline Clínico reparativa & 7 & 10 & 17 & 19 & 24 & 21 & 35 & 21 & 18 & 172 & 57.9 \\
\hline Epidemiológico & 2 & 5 & 4 & 5 & 8 & 4 & 8 & 12 & 7 & 55 & 18,5 \\
\hline Medicina Preventiva y S. $\left({ }^{*}\right)$ & 1 & 1 & 2 & 2 & 5 & 2 & 4 & 2 & 1 & 20 & 6,7 \\
\hline Tecnologías aplicadas (**) & - & - & - & - & - & - & - & 1 & - & 1 & 0,3 \\
\hline Cjencias Básicas & 1 & 1 & 3 & 5 & 2 & 2 & 8 & 5 & 4 & $3 !$ & 10,4 \\
\hline Educación Médica & - & - & 1 & - & - & - & 1 & $\therefore$ & - & 2 & 0,7 \\
\hline Etno-medicina & $\cdot$ & 1 & - & - & 3 & - & 2 & - & - & 6 & 2,0 \\
\hline Historia de la Medicina & - & 1 & 1 & - & 1 & 1 & 2 & - & - & 6 & 2,0 \\
\hline Economía en Salud & - & - & 2 & - & - & - & - & - & - & 2 & 0.7 \\
\hline Etica y Deontología Médjea & - & - & - & - & $\cdot$ & 1 & - & - & - & 1 & 0,3 \\
\hline Salud Ambiental & - & 1 & - & - & - & - & - & - & - & 1 & 0,3 \\
\hline Total & 11 & 20 & 30 & 31 & 43 & 31 & 60 & 41 & 30 & 297 & 100,0 \\
\hline
\end{tabular}

(*) Medicina Preventiva y Sảud Publica

(**) Tecnologías aplicadas al diagnóstico y tratamiento 
En las revistas biomédicas nacionales se aprecia diferentes patrones en la periodicidad de presentación de los temas (Tabla $N^{\circ} 13$ ). Así, algunos temas como el cáncer, tuberculosis y salud mental se han mantenido presentes a través de todo el período como uno de los cinco primeros temas motivo de publicación. Otro patrón lo conforman temas que no eran tomados en cuenta y de pronto aparecen en los últimos 3 años entre los primeros cinoo temas, tal es el caso de el cólera $y$ las enfermedades metaxénicas. Un tercer patrón es aquel en que aparecen los temas esporádicamente, como salud reproductiva y desnutrición. Un úlimo patrón es aquel en que un tema apareció como uno de los cinco primeros y desapareció bruscamente como el SIDA y las enfermedades del colágeno. En las revistas biomédicas extranjeras hay algunas variaciones (Tabla $\mathrm{N}^{\circ} 14$ ).

\section{DISCUSIÓN}

Respecto a las publicaciones nacionales, los resultados muestran que en el periodo de estudio se han caracterizado por su irregularidad. Son pocas las revistas biomédicas en las que se observó una tendencia a la regularidad, característica que sobre todo se dio en las revistas relacionadas a Sociedades Científicas. Este hecho muestra la importancia de estas organizaciones para el desarrollo de la actividad científica; pero también es posible que guarde relación con el manejo administrati- vo de la edición de la publicación, que en otras instituciones como los Ministerios o las Universidades son más complejos e impersonales.

En términos del volumen de artículos publicanos se observa entre el inicio y el final del periodo un discreto aumento del volumen de publicaciones $(12 \%)$. Si se toma como referencia un periodo nayor, se puede observar un incremento de 157 publicaciones en $1979\left({ }^{4}\right)$ a 311 en 1993, es decir, que en un peniodo de 15 âños, el número de publicaciones biomédicas aumento en $98 \%$. Este incremento es insignificante si se compara con otros países de la región. México, el segundo país de mayor producción científica en América Latina regsstró un incremento del volumen de artículos publicadios entre 1980-1989 de un 66\% (3).

El marcddo incremento en el volumen de artículos en 1991, con un 32,2\% respecto al año anterior, está en clara relación con la epidemia del Cólera. Aparentemente, ello puede ser un indicador del criterio de oportunị̣ad que prima en la realización de investigaciones, toda vez que a partit del año siguiente se observa un descenso sostenido. Es posible, de acuerdo a los datos presentados por Gonzáles Mugaburu, que haya ocurrido una situación similar en 1981, año de la epidemia de Tifoidea en Lima Metropolitana. donde se observa un incremento en el número de estudios del $13 \%$ respecto al año anterior, lo cual es significativo, teniendo en cuenta el limitado número de revistas medicas en circulación durante dicho periodo ( $\left.{ }^{4}\right)$.

TABLA 13.- Cinco primetos temas* motivo de publicación en revistas biomédicas nacionales. Perú, 1985 - 1993.

\begin{tabular}{|c|c|c|c|c|c|c|c|c|c|c|}
\hline Temas & $\mathrm{N}^{0 \cdot}$ & 1985 & 1986 & 1987 & 1988 & 1989 & 1990 & 1991 & 1992 & 1993 \\
\hline Farmacología Clínica & 160 & & & 150 & & $2 \mathrm{do}$ & lio & 200 & 1ro & 1ra \\
\hline Cáncer & 150 & 410 & 1 ro & 410 & 1 to & 110 & 2 do & & & tro \\
\hline Enf. Gíneco-Obstétricas & 120 & 3 ro & 350 & 350 & 350 & 50 & & & 4 to & \\
\hline Enf. del Aparato Digestivo & 113 & 1 ro & & 3 ro & & & $4 t o$ & Sto & 3ro & \\
\hline Enf. Neurológicas & 91 & & & Sto & 3 ro & 410 & 510 & & & 3 ro \\
\hline Patología Oftalmológica & 87 & 4 to & 4 to & 350 & 2 do & & & & & \\
\hline Microbiología y Parastcología & 84 & 5 to. & 5 to & & 510 & & & & & $2 d 0$ \\
\hline Tuberculosis & 75 & & & & & & & 3ro & & \\
\hline Salud Mental & 145 & $2 d 0$ & 2 do & $2 \mathrm{do}$ & 350 & 3 ro & 310 & 4 to & $4 t 0$ & \\
\hline Fármaco-dependencia & 85 & & & $2 d o$ & 350 & & & & & \\
\hline Enf. del Metabolismo & 63 & & & & & & & & $2 d 0$. & \\
\hline Cólera & 61 & & & & & & & lro & & \\
\hline Enf. Metaxénicas & 50 & & & & & & & & 5 to & 4 to \\
\hline Medicamentos y Salud Príblica & 47 & & & & & & & & $4: 0$ & \\
\hline Zoonosis & 39 & & & & & & & & & 4 to \\
\hline Desnutrición & 58 & & & & 410 & & & & & 5 to \\
\hline Enf. por animales ponzoñosos & 26 & & & 4 to & & & & & & \\
\hline
\end{tabular}

* La ubicación se estableció según la frecuencia anual de artf́culos publicados, por lo gue en algunos años hay temas que tienen la misma ubicación.

* Número total de artículos publicados en el periodo de estudio. 
TABLA 14.- Cinco primeros temas* motivo de publicación en revistas biomédicas Extranjeras. Peru, 1985 - 1993.

\begin{tabular}{|c|c|c|c|c|c|c|c|c|c|c|}
\hline Temas & $N^{a * *}$ & 1985 & 1986 & 1987 & 1988 & 1989 & 1990 & 1991 & 1992 & 1993 \\
\hline Microbiología y Parasitologia & 21 & ler & $2 \mathrm{do}$ & & $3 \mathrm{er}$ & & 410 & 2 do & ler & 410 \\
\hline Enfermedad Diarrejca aguda & 36 & $2 \mathrm{do}$ & 1er & $2 \mathrm{do}$ & $3 \mathrm{er}$ & ler & & $1 \varepsilon r$ & $2 \mathrm{do}$ & 2 do \\
\hline Câncer & 18 & 2do & $3 \mathrm{er}$ & 4to & & 410 & 1ro & 410 & 3 ro & $2 \mathrm{do}$ \\
\hline Enfermedades Metaxénicas & 22 & 2 do & $3 \mathrm{er}$ & 2do. & $3 \mathrm{er}$ & & 2do & 410 & 2do & ler \\
\hline Desnutrición & 34 & $2 \mathrm{do}$ & $2 \mathrm{do}$ & ler & ler & $3 \mathrm{er}$ & 2do & 3 ro & 1er & 3 ro \\
\hline Enfermedades Prevenibles por vacunas & 2 & $2 \mathrm{do}$ & 3er & & & & & & & \\
\hline Atención Primaria & 1 & $2 \mathrm{do}$ & & & & & & & & \\
\hline Medicita Tradicional & 7 & & $2 \mathrm{do}$ & 410 & & 5 to & & 5 to & & \\
\hline SIDA & 7 & & $3 e r$ & & 3er & & & 410 & & \\
\hline Salud Mental & 4 & & 3er. & 3er & & & & & & \\
\hline Salud Reproductiva & 7 & & $3 e r$ & & . & 40 & & & 310 & 410 \\
\hline Hepatitis Viral & 5 & & $3 \mathrm{er}$ & & & & 410 & & 3 ro & \\
\hline Patología Oftalmologica & 2 & & $3 e r$ & & & & 410 & & & \\
\hline Zoonosis & 12 & & & $2 \mathrm{do}$ & 410 & & 3 ro & & $2 \mathrm{do}$ & 3 ro \\
\hline Farmacología Clínica & 15 & & & 3er & $2 \mathrm{do}$ & $3 \mathrm{er}$ & $3 \mathrm{ro}$ & 510 & & 3ro \\
\hline Enfermedades Reurnatológicas & 2 & & & 460 & 4 to & & & 410 & & \\
\hline Enf. transmitidas por animales ponzoñosos & 7 & & & 410 & 4 to & & & 410 & 3 ro & \\
\hline Patologia de Altura & 19 & & & & $3 \mathrm{er}$ & 5 to & $3 r 0$ & $2 \mathrm{do}$ & ler & ler \\
\hline Enfermedades Dermatológicas & 21 & & & & 3er & & 410 & & & \\
\hline Cólera & 6 & & & & 410 & & & & $2 \mathrm{do}$ & 3 ro \\
\hline Fisiología de la Reproducción & 9 & & & & 4to & $2 \mathrm{do}$ & & & $3 r 0$ & 4 to \\
\hline Tuberculosis & 2 & & & & & & 410 & & 3 ro & \\
\hline Enfermedades Neurológicas & 3 & & & & & & 4 to & & & \\
\hline Farmaco-dependencia & 5 & & & & & & & & $2 \mathrm{do}$ & 410 \\
\hline Enfermedades Gíneco-Obstétricas & 1 & & & & & & & & $3 \mathrm{ro}$ & \\
\hline Políticas en Medicamentos & & & & & & & & & 3 ro & \\
\hline Enfermedades Genéticas & 3 & & & & & & & & 3 ro & 410 \\
\hline Enfermedades del Aparato Digestivo & 3 & & & & & & & & 3 ro & \\
\hline
\end{tabular}

* La ubicación se establecío según la frecuencia anual de artículos publicados; por lo que en algunos años hay temas que tienen la misma ubicación.

* Número total de artículos publicados en el periodo de estudio.

De otro lado se observa un número cada vez más creciente de revistas extranjeras que publican estudios realizados en el Perr. Ello puede significar un mayor interes de los investigadores nacionales por dar a conocer su producción en publicaciones del exterior. Es posible que un componente de dicha motivación esté relacionado con las exigencias de las instituciones que financian las investigaciones. Sin embargo, es importante señalar la ventaja potencial que plantea la publicación en una revista internacional: mayor difusión de los hallazgos, posibilidad de contactarse con otros equipos de investigación en el área y la posibilidad de ser un referente en el tema para investigadores extranjeros $y$ agencias o fundaciones interesadas en desarrollar o finaneiar investigaciones en la region. Un ejemplo claro de ello se observa en un análisis cuantitativo de la literatura cientifica de América Latina realizado por Garfield. Este encuentra que en el periodo 1981-1993, la producción cientffica 
peruana registrada en el Science Citation Index (SCI) ocupó el 8\%o. lugar en la región. Un anátisis por áreas encontró que a pesar de tener el 8vo. lugar en Ciencias Biológicas y en Medicina Clínica, las publicaciones peruanas tuvieron uno de los mayores f́ndices de impacto $(6,31$ y 6,41 citas por artículo respectiyamente), superando incluso a países con un mayor valumen de producción científica como Chile, Venezuela y Cuba $\left({ }^{*}\right)$. Ello es un indicador de que las investigaciones nacionales publicadas en revistas extranjeras constituyen uno de los núcleos más importantes en términos de complejidad, cumplimiento de los estándares de publicación internacionales, oportnnidad científica y originalidad de la producción cientffica nacional. Este al to nivel de impacto mundial se contrasta con el aparente poco impacto que tiene la producción cientifica nacional a nivel Latinoamericano. Así tenemos que para el periodo 1986-1990, los artículos peruanos representan el $0.9 \%$ de las citas de todos los articulos publicadas en el Boletín de la OPS ( ${ }^{3}$ ), lo cual sería un indicador de que la producción científica nacional de mayor complejidad esta claramente orientada a su publicación y circulación en publicaciones de circulación mundial.

Si se asume que las investigaciones nacionales de mayor complejidad y trascendencia son las que alcanzan a ser publicadas por revistas internacionales, y si se acepta el hecho del poco acceso que los médicos del medio tienen a estas publicaciones ("), se estaría asistiendo a una difusión cada vez menor de los resultados de dichas investigaciones en el país, lo cual constituye un obstáculo importante para la confrontación de experiencias dentro de la colectividad científica nacional. A su ve $z_{\text {, este }}$ filtimo hecho va afectar de manera importante al dinamismo de la actividad científica peruana.

Por otro lado existen indicios de que ha existido cierta vasiabilidad en la proporción de investigaciones sobre el Perú publicadas en el extranjero. González Mugaburu describe un $4,8 \%$ de trabajos publicados en el exterior para 1980 y $7,2 \%$ para 1981, siendo esta última cifra superior a la proporción que se observa en los dos primeras años estudiado en el presente estudio. El brusco aumento de dicha proporcion durante 1991, año de la epidemia del Cólera y su posterior declinación. (Fig. $N^{\circ} 1$ ), puede ser muestra de una endeble relación con la colectividad cientifica extranjera, mediada sobre todo por un sentido de oportunidad.

En lo referente a los autores, se observa una tendencia a la producción individual. El porcentaje de estudios con un autor legó en 1993 a un $45,6 \%$ de los estudios publicados en el país en dicho año. Este hecho es indicativo de un tipo de práctica científica que en el mundo está cambiando. Hoy, dada la complejidad y los costos de las investigaciones, se tiende a la formación de grupos y organizaciones dedicadas a la investigación, antes que al ejercicio individual. Por ejemplo García observó en 1978 al analizat las investigaciones publicadas en Cuba, que sólo el 17,8\% de ellas tenjan un solo autor, lo que tomo como un indicador del carácter colectivo del desarrollo de la investigación en dicho país ("). También pue- de ser reflejo del tipo de investigación que se bace en el país, tanto por los temas que trata, los enfoques que adopta y la calidad de sus resultados. Finalmente no hay que descartar la posibilidad de que también sea expresión de las relaciones sociáles, económicas y culturales del país, donde la pequeña empresa, el trabajo informal y el artesano florecen día a día. Sería necesario hacer un análisis más exhaustivo de estos aspectos, a fin de encontrar las causas de este fenómeno y promover la investigación en el sentido que posibilite el máximo desarrollo, pero sin contradecir lo que hay de genuino y creativo en la actividad intelectual peruana.

La paca presencia de autores extranjeros, tanto en publicaciones nacionales como extranjeras, muestra una débil influencia de científicos de otros países en la producción cientifica nacional en el periodo estudiado. Los que mayor presencia han tenido fueron investigadores de Estados Unidos, país al que constantemente migran médicos egresados de las universidades peruanas ( 10 ).

También se observó una poca participación de autores residentes en provincias, lo cual es compatible con lo que se observa en otros países de la región ( $\left.{ }^{12}\right)$. La distribución porcentual de los autores principales con residencia en provincias mostró que los cinco primeros lugares (en un $74 \%$ ), correspondieron a departamentos donde funcionan Facultades de Medicina. Este becho confirma lo que en diversas oportunidades se ha señalado, respecto al rol dinamizador de las universidades jespecto al desarrollo de la investigación en dichas regiones.

Otro hecho importante es el incremento de la participación de profesionales no médicos en las publicaciones biomédicas nacionales, que en 1993 alcanzó un 12,9\% de los estudios publicados. Es importante destacar que dicha tendencia ascendente se da desde 1991. Es muy posible que problemas de salud sumamente complejos, como la epidemia de Cólera, sean la causa de esta tendencià, en la medida que su investigación plantea la necesidad de un enfoque multidisciplinario

Respecto a los tipos de publicaciones, se observa que el número de artículos originales en las revistas nacionales decrece en favor de otros tipos de trabajos, lo cual puede ser expresión de un menor rigor en la selección de las contribuciones o del "carácter" de las publicaciones nacionales. En cambio, en revistas biomédicas extranjeras los tipos de contribución que más se publican son la producción original, las comunicaciones breves y las cartas al editor, escritos que en su mayoría representan una contribución importante al conocimiento y la discusión de los problemas científicos mảs importantes.

Un hallazgo que es importante analizar en sus implicancias es la escasa publicación de "Cartas al Editot" en revistas biomédicas nacionales. Esto puede ser la expresión de una situación que caracteriza al ambiente intelectual nacional: la falta de diálogo. Conducta contraria al desarrollo cientifico, pues desalienta a los autores, no estimula el intercambio de jdeas ni e] análisis crítico de los resultados. De este modo, las observaciones de los investigadores quedan olvidadas y peligrosamente 
consagradas por el silencio de la colectividad científica nacional.

Las áreas temáticas muestran la orientación de los autores y editores. En este estudio se observó que mientras las revistas biomedicas nacionales publicaron con mayor frecuencia artículos clínicos; en las revistas biomédicas extranjeras se publicaron en una buena proporción estudios de epidemiología y ciencias básicas; esta tendencia, sobre todo lo referente a las ciencias básicas, se ba venido observando en otros países de la sub-región (19: 44$)$. Esto es realmente sorprendente, en la medida que por un lado expresa la calidad de los estudios y por otro las areas en las que los peruanos pueden hacer mejores contribuciones al desarrollo científico mundial.

Un hecho que llama poderosamente la atención es la baja proporción de investigaciones en el área de Medicina Preventiva y Salud Pública. De acuerdo con un estudio realizado por Domínguez, para 1988 existían 556 investigaciones, en su mayoría del área de Medicina Preventiva y Salud Pública, ejecutadas, en proceso de ejecución o en proyecto, lo cual hacía de esta área uno de los núcleos más importantes de desarrollo de investigación en salud en el país (15). Sin embargo, en el período 1985-1993 sólo se publicaron 176 trabajos de ésta área. Esta baja proporción de investigaciones seria un indicador de la poca difusión que tienen los resultados de estos estudios a través de las publicaciones biomédicas del país. También cabe la posibilidad que exprese un desarrollo poco formal del proceso de investigación, en el cual a la etapa final: la publicación científica de los hallazgos, no se le de la importancia debida; con lo cual se reducen las posibilidades de utilización e impacto de estos estudios $\left({ }^{16}\right)$.

El análisjs de los cinco primeros temas de cada año, según frecuencia de artículos, nos muestra que han habido cambios importantes respecto a los temas que se estudian. Entre las probables causas se pueden señalar las siguientes:

La influencia de "modas cientificas". Un elemento que llama la atención es la Farmacología Clínica como uno de los principales temas de investigación sobre todo en el periodo 1987-1993. Ello muestra que el mayor porcentaje de la actividad en investigación en salud pasa por estudios de medicamentos, los cuales en muchos casos son promovidos por los laborato. rios, los cuales generan estas "modas cientificas" como estrategia de mercadeo de sus productos $\left({ }^{17}\right)$. Prueba de ello, es el hecho que la proporción y número de artículos con dicha temática no alcance proporciones semejantes en los trabajos sobre el Perú publicados en el extranjero. De otro lado, el desartollo de esta temática en nuestro medio hace que no se aprecie en gran magnitud, otras "modas científicas" como el caso del SIDA que han orientado y contribuido significativamente a la producción cientifica de otros países en desarrollo ( ${ }^{\text {IB }}$ ).

Criterios de oportunidad. En algunas circunstancias el in- teres de la colectividad cientifica nacional o internacional por un tema especffico da mayor oportunidad a los investigadores para estudiar y publicar. Es probable que en estos casos ocura una mayor oferta para desarrollar investigaciones, mejores condiciones para el trabajo conjunto con otros equipos de investigación nacionales o extranjeros, una mayor avidez por publicar todo lo que se haga respecto al tema o que en última instancia lo novedoso sea una importante motivación para el desarrollo de investigaciones. Una muestra de este criterio de oportunidad es el Cólera, el cual aparece como unos de los temas más frecuentes en 1991, en trabajos publicados en el país como en el extranjero a partir del año 1992; observándose en muchos casos una autoría conjunta de investigadores nacionales con grupos de investigación foráneos. Pero, para el año 1992 ya no figura en los cinco primeros lugares de los temas publicados en las revistas biomédicas nacionales, a pesar de continuar siendo uno de los principales problemas de salud pública del país.

Disponibilidad de recursos para la investigación en algunos sectores. Esto ha ocurrido en algunas instituciones que cuentan con recursos materiales y humanos suficientes para mantener una fuerte y sostenida producción en algunas líneas de investigación, como en el caso de los estudios acerca del cáncer o medicina de altura.

La presión sacial. Ello se observó en el caso de] Cólera, que en 1991 produjo un incremento muy importante de artículos y ocupó el primer lugar en la producción por área temática. El cólera además impuso un estilo de investigación más integral, con participación de profesionales de las áreas de ciencias sociales y humanas. Decididamente, las épocas de epidemia tienen la virtud de mostrar, sin recato, la naturaleza social de los problemas de salud, por ello es que es tan fácil que en estas circunstancias los especialistas incluso se hagan políticos.

La transición epidemiológica. La importante proporción y continuidad de temas como el Cáncer, Salud Mental, Fármacodependencia, Enfermedades Neurológicas, etc. puede ser consecuencia de la influencia de la transición epidemiológica del país, que en el caso del Perú es expresión de un perfil de morbi-mortalidad mixto donde coexisten en proporciones importantes las enfermedades infecciosas y las enfermedades crónico-degeverativas $\left({ }^{19}\right)$.

El rol promotor de las sociedades cientificas. La presencia permanente en proporciones importantes de temas como las Enfermedades del Aparato Digestivo, las Enfermedades Neuropsiquiátricas, Las Enfermedades Gíneco-Obstétricas y Epidemiologfa, así como la continuidad de las publicaciones de las sociedades cientrficas, se debe al rol dinamizador que estas instituciones tienen en nuestro medio en aspectos vinculados a la investigación, discusión cientifica y publicación, tal como se señala en el estudio del Dr. Javier Mariátegui acerca de la evolución de la historia societaria de la Psiquiatría en el Perí $\left({ }^{20}\right)$. 
¿Cómo explicar que cerca al año 2,000 las socjedades cientificas pueden seguir marcando el paso de la investigación biomédica en el país, de manera similar a lo observado en las primeras décadas de este siglo?. La respuesta podría sel que en el Perú, como en otros paîses del área, las instituciones que pertenecen al campo de la salud no están orgánicamente articuladas, hay una multiplicidad de centros de decisión, no hay flujo de información entre ellas y no se persiguen objetivos comunes. El resultado es la falta de coordinación, el conflicto, la superposición y el paralelismo de las acciones lo que lleva a una baja productividad general y un relativo impacto de las instituciones del campo de la salud frente a los procesos que determinan las tendencias de la aclividad cientifica en nuestro medio $\left(^{(1)}\right)$

La crisis de la universidad peruana. Por lo visto anteriormente existió una débil infuencia de la universidad en el establecimiento y desarrollo de las tendencias en las diferentes lineas de investigación. Si bien en la década de los ochenta existieron diversos problemas de tipo social, una grave recesión económica y una grape crisis del sistema cducativo universitario que podrían explicar la falta de capacidad de respuesta de la universidad frente a las tendencias de desarrollo en la investigación biomédica; pareciera que el nival académico lam. bién la jugado un rol importante en la aparente marginalidad de la unjversidad respecto a la actividad científjca nacional. La critica expresada por Bronner al referirse a la actividad intelectual de las universidades latinoanericanas describe esta realidad, aunque no la explica: "En muchas de ellas la calidad de la vida intelectual que desarrollan se ha venido al suelo o no ha logrado despegar nunca. Los académicos producen poco o lo hacen de maneras poco relevantes para la sociedad; los cursos que se imparten son obsoletos y aburridos; la vida departamental es muchas veces un remedo de trabajo que se supone que debe realizar un equipo intelectual. Es una crisis de autoridad intelectual en la propia institución universitaria." (22).

La existencia de núcleos de modernidad en la colectividad cientifica nacional. El análisis de los principales temas publicados en el extranjero nos muestra una gran continuidad, lo cual es un indicador de la presencia de grupos de investigadotes que desarrollan líneas permanentes de estudio. Es posible que estos grupos, con un número importante de publicaciones en el extranjero representen el sector de investigadores que han alcanzado un mayor nivel de desarrollo en nuestro pais, tanto a nivel de originalidad de sus aportes, como de una sistemática y organización del trabajo científico compatibles con los desarrollados en las instituciones de investigación de los países centrales. Sin embargo, el estudio de su producción temática en función del total de los estudios publicados, muestra que su número y producción es minoritaria, lo que expresa en principio su poca capacidad para ejercer un liderazgo que influcncie en la actividad cientifica nacional y de otro lado una orientación de su producción preferentemente a la publicación en el extranjero como eje de su actividad en investigación.
Finalmente, todo to descrito en este estudio no hace sino mostrar una yariación constante en la producción científica nacional, orientada más por impulsos que por una política definida de desartollo cjentífico-tecnológico. No se puede juzgat cuán positiva o negativa es esta forma de hacer ciencia; pero analizada desde la perspectiva de la formación de grupos de investigación, del perfeccionamiento en ciertas áreas basta el punto que sean considerados referentes internacionales y de una real contribución a la solución de los pioblemas del pais, no cabe duda que se esta lejos del nivel que se esperaría de un país con importantes riquezas naturales y un singular potencial humamo. De otro lado, los hechos descritos, si bien tienen relación con la pobreza econónica del país, también eșán en relación con la falta de persistencia y continuidad en el trabajo, la ausencia de consensos y compromisos colectivos y la carencia de una visión a futuro. Es probable que estos sean los mayores obstáculos para el crecimiento y desarrollo de la ciencia en el Perú.

\section{BIBLIOGRAFIA}

1) Organización Panamericana de la Salnd. La investigación en salud en America Latiba: Estudio de países seleccionados, Pub. Cjent 543 Waskington D.C., 1992.

2) Euber G, La entrega de docusentos enl los países en desarrollo y cuestiones tecinológicas afmes. Educ. Méd Salud, 1981, 15:442-453.

3) Webbr R, Feriández Baca G. Perí en Námeros 1991. Anuario Estadístico.

4) CONCYTEC, MINSA. Políticas de Investigación Científica en Salud en el Perú. Areas promtarias y estrategias., Lima 1984. pag 76.

5) Estrada C, Aurlesen J, Villar A et al. Tipos de estudios epidemiológicos en revistas biomédicas peruanas. Diagnóstico, 1988, (21): $166-169$

6) Cneto M. Excelencia cientifica en la periferia. Actividades cientíEicas e iavestiqación biomédica en el Perí 1890 - 1950. GRADE CONCYTEC, 1989

7) Taba BG, Toussaint CS, Borges YA, Vieyra AA. La producción científica en México, 1980. La Investigación en Salud en America Latina. OPS, Washington 1992, pag $93-118$.

8) Garfield E. Análisis cuantitativo de la literatura cientifica y sus repercusiones en la formulación de políticas cientificas en América latina y el Caribe, Boletín de la O.S.P., 1995, 118:448-456.

9) Silva L, Pino A, Miranda Z, Martíncz V, Pérez C. Origen nacional de los artículos publicados o citados en el Boletín de la OSP de 1971 a 1990. Boletín de la O.S.P., 1994, 116:331-337.

10) Alarcón J. y Colaboradores. Situación de trabajo y características socioeconómicas del médico de Lima Metropolitana. Limà, Calegio Médico del Perú, 1999

11) García DI, Espinoza CL, Estevez JM, Bordon GJ, Bordon GM. Revistas Cubanas Médicas. Un estudio de gestión sistémica en la tama de salud. Revista Cubana de Administración en Salud. 1981. 7: $294 \cdot 314$.

12) De la Fuerte J, Pedrón N, De León C, Salinas V. Análisis de la prodnccion cientifica nacional en Biomedicina y Salud 1985-1987. Gaceta Médica de México, 1990, 126:267-274.

13) De Souza C, Costa LJ, Schnitt C, Piegas M, Peixinho A, Schnidd A, Briquel A. Publicaçoes biomédicas brasileicas na literatura cientófica intenacional. Doenças Endêmicas transntissíveis. Educ. Med. Salud, 1986, 20:72-83. 
14) Alarcón SA. Panorama de la investigación biomédica en México. Comparación entre las décadas 1968-1977 y 1978-1987. Gaceta Médica de México. 1990, 126:274-282.

15) Domínguez del Río G. Instituciones, Investigadores e investigaciones en salud del Perú. INDES, Lima 1989, pagg 26-32.

16) Kumate J. Necesidades de información en Ia investigación biomedica. Edacaciớn Médica en Salnd, 1981, 15: pags. 395-405

17) Briquet de Lemos A. Infraestructura da literatura biomédica: copsideraçoes acerca de um núcleo de revistas brasileiras do setor saúde. Educación Médica y Salud, 1981, 15: pags. 406-423.
18) Elford J, Bor R, Summers P, Research into HIV and AIDS between 1981 and 1990: the epidenic curve. AIDS, 1991, 5: 1515-1519.

19) Frenk J, Frejka T, Bobadilla J, et al. La transición epidemiológica en Anérica Latina. Boletín de la OPS, 1991, 111:485-496.

20) Mariategui J. Historia Societaria de la Psiquiatría en el Perú. Revista de Neura-Psiquiatria, 1987, 50: 12-23.

21) Pellegrini FA. Organización de la actividad científica en salud: problenas y perspectivas. Educ. Med. Salud, 1987. 21:185-192.

22) Brunger, citado por Portillo J. La Salud en América Latiua: una aproximación. Ciencias Sociales y Medicina. La Salud en LatinoAmérica. Inst. de lov. Facultad de CC. \$S., Buenos Aires 19y4, p. 37. 